\title{
Local relaminarization mechanism induced by a dynamic free-slip boundary
}

\author{
Cong Wang @ * and Morteza Gharib \\ Division of Engineering and Applied Science, California Institute of Technology, \\ Pasadena, California 91125, USA
}

(Received 26 April 2021; accepted 6 July 2021; published 12 August 2021)

\begin{abstract}
Applying a dynamic free-slip boundary in a turbulent boundary layer has been shown recently to shift outward the near-wall transverse vorticity away from the wall and reduces the wall skin friction by more than $40 \%$. Herein we present a local relaminarization mechanism induced by the dynamic free-slip boundary, from the perspective of energy exchange and transportation. The spatial evolution of the energy components associated with the mean motion, turbulent motion, and a shear-free oscillatory motion is presented. An analysis of the average energy exchange process in the near-wall region suggests that the energy of turbulence is transferred to the mean motion, against the canonical downward turbulent energy cascade. A considerable amount of energy is supplied to the shear-free motions, which displaces the highly turbulent and shear motions away from the wall. The relaminarization mechanism is associated with outward-shifted transverse vorticity and the depletion of the shear motions near the wall. As an effective method to manipulate the critical region for wall shear stress generation, the dynamic free-slip boundary produces a much stronger effect than the conventional relaminarization process, which can be employed for efficient drag reduction and boundary layer control.
\end{abstract}

DOI: 10.1103/PhysRevFluids.6.084604

\section{INTRODUCTION}

Wall-bounded turbulence remains one of the least understood phenomena in fluid mechanics, despite its significance in many engineering fields. For example, approximately $50 \%-80 \%$ of the total energy expenditure of commercial airplanes and container ships is used to overcome the turbulent frictional drag [1,2]. The reason for the low-energy efficiency is because the turbulent motions generate large skin friction on the surface of water or air vehicle and dissipate a significant amount of energy through the downward turbulent energy cascade [3]. In contrast, a relaminarized turbulent boundary layer (TBL) generates much reduced wall shear stress [4], which is of great interest to both scientists and engineers. Turbulent boundary layers are commonly relaminarized when imposed with a strong favorable pressure gradient [5-8]. The favorable pressure gradient accelerates the bulk flow forward, which stabilizes the TBL by reducing the turbulent production while increasing the turbulent dissipation. Consequently, the wall shear stress drops over a certain developing distance. However, although such a global pressure condition can be created in the laboratory (usually generated by reducing the cross section of the wind tunnel or water tunnel), it is difficult to implement over the surface of ships and airplanes. In addition, accelerating the bulk flow is not energy efficient, even though the relaminarized turbulent flow creates less wall skin friction.

To reduce the skin friction of wall-bounded turbulent flows, we recently invented a dynamic free-slip boundary method [9], which can be easily implemented on the surface of water vehicles

*cwwang@caltech.edu 
(a)

$$
\text { Mean motion } \frac{-\left(\overline{u_{i}^{\prime} u_{j}^{\prime}}\right) \frac{\partial \overline{U_{i}}}{\partial x_{j}}}{\mathbf{T K P}} \longrightarrow \text { Turbulent motion }
$$

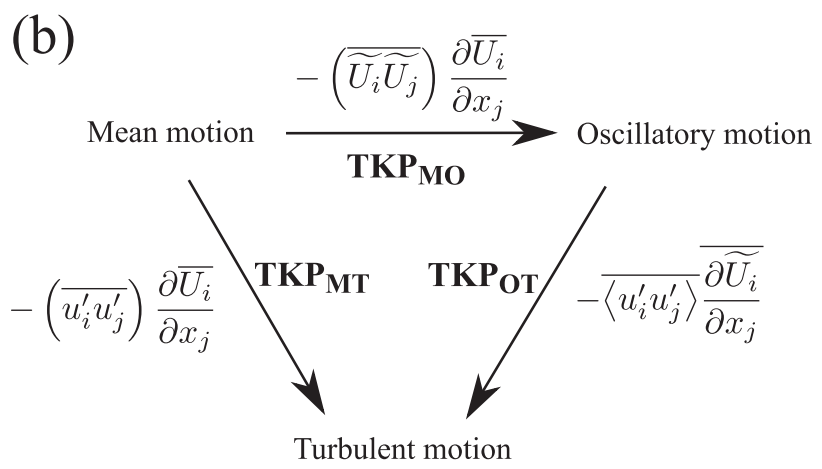

FIG. 1. Analytical expressions representing the energy exchange process with (a) Reynolds decomposition and (b) triple decomposition. The $\langle\cdot\rangle$ represents the phase average. The arrows indicate the direction of energy transfer.

to achieve significant wall shear stress reduction (by more than $40 \%$ ). The details on creating the dynamic free-slip boundary can be found in [9]. Due to the periodic perturbation of the dynamic free-slip boundary, the TBL velocity field can be triple decomposed as $U_{i}=\bar{U}_{i}+\widetilde{U}_{i}+u_{i}^{\prime}$ instead of the conventional Reynolds decomposition $U_{i}=\bar{U}_{i}+u_{i}^{\prime}$, where $\bar{U}$ is the time-averaged velocity, $u_{i}^{\prime}$ is the residual turbulent fluctuation, and $\widetilde{U}_{i}$ is the coherent oscillatory motion that is phase locked to the dynamic oscillating surface [10]. Energy exchange processes happen within the decomposed velocity components and the complete energy equations are given in Appendix A. With Reynolds decomposition, the energy exchange happens between the mean motion $\bar{U}$ and the turbulent motion $u_{i}^{\prime}$, which is often referred to as the turbulent kinetic energy production (TKP) [given in Fig. 1(a)]. In typical turbulent shear flows, TKP is positive, representing an energy supply to turbulence through the downward energy cascade. With triple decomposition, energy transfers among the three velocity components, which are denoted by $\mathrm{TKP}_{\mathrm{MT}}$, $\mathrm{TKP}_{\mathrm{MO}}$, and $\mathrm{TKP}_{\mathrm{OT}}$ in Fig. $1(\mathrm{~b})$, where $\mathrm{M}, \mathrm{O}$, and $\mathrm{T}$ represent the mean motion, the oscillatory motion, and the turbulent motion, respectively.

In this paper we report the discovery of a local relaminarization mechanism in the most critical region for wall shear stress generation. The remainder of this paper is organized as follows. Some information about the experiment is given in Sec. II. In Sec. III the spatial evolution of energy components, the detailed energy exchange processes, and the critical velocity gradient distribution is presented. A discussion that links the relaminarization mechanism to the TBL structural changes (presented in [9]) is given in Sec. IV. A summary is given in Sec. V.

\section{EXPERIMENTAL SETUP}

The experimental conditions are the same as in [9] and the velocity field in the central line region of the dynamic boundary array is quantitatively mapped by high-speed digital particle image velocimetry (DPIV). The dynamic boundaries are set to protrude into the TBL and are modulated in phase by a $50-\mathrm{Hz}$ signal, with a maximum height oscillating within the range of $0.8-2.4 \mathrm{~mm}$. 
(a)

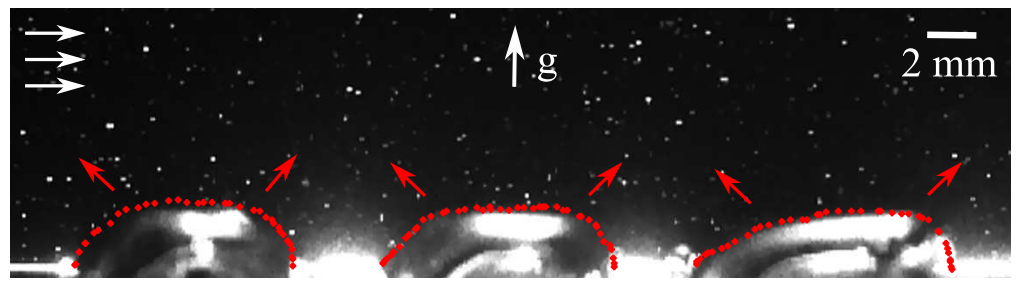

(b)

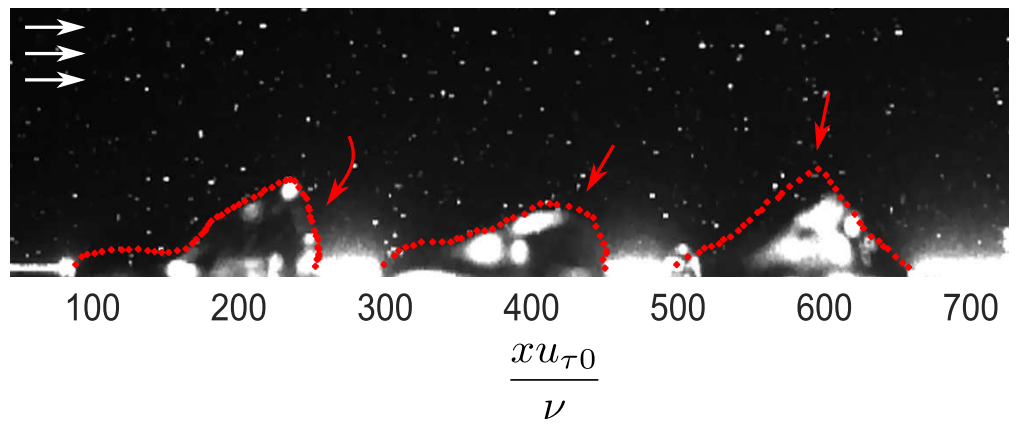

FIG. 2. Snapshots of the (a) expanding and (b) contracting dynamic free-slip boundaries. The air-water interface is highlighted by red dotted lines and the movement direction of the interface is qualitatively shown by the arrows. The normalized $x$ coordinate $\left(\frac{x u_{\tau} 0}{v}\right)$ is shown along the bottom edge, which will be referred to for presenting the results.

The nondimensional height $\left(h_{0}^{+}=\frac{h u_{\tau 0}}{v}\right.$, where $u_{\tau 0}$ is the frictional velocity of the baseline flat plate TBL) varies between 16 and 48 . Although the dynamic surfaces generally oscillate in phase, as an extremely compliant surface, they are sensitive to the local turbulent fluctuations. Figures 2(a) and 2(b) show two snapshots of the expanding and contracting free-slip boundaries. The dynamic oscillation is highly nonlinear and nonsymmetric. When in expansion motion, the curvature of the dynamic surfaces is approximately uniform. When in contraction motion, however, sharp local curvatures are observed. As will be discussed in Sec. IV, the dynamic curvature may be the fundamental driving force of the relaminarization mechanism. Figure 2 also gives the streamwise coordinate that will be employed for presenting the results.

\section{RESULTS}

\section{A. Spatial distribution change of the energy components}

The magnitude of $\bar{U}, \widetilde{U}$, and $\overline{u^{\prime}}$ reflects the respective amount of associated energy. Figure 3(a) shows the profiles of the mean streamwise velocity $\bar{U}$ in the upstream and downstream regions of the dynamic boundaries (at $\frac{x u_{\tau 0}}{v}=80$ and 660 in Fig. 2). When compared with the baseline case (the flat plate TBL case, shown by the black solid line), $\bar{U}$ significantly decreases in the near-wall region, starting from the upstream region. At both locations, strong oscillatory motion $\widetilde{U}$ is generated, as shown by the phase-averaged profiles in Figs. 3(b) and 3(c). The oscillatory motion is more energetic in the downstream region, with its magnitude up to $10 u_{\tau 0}$. Interestingly, as was reported in [9], $\widetilde{U}$ generates zero wall shear stress on average because of its symmetry. As such, the energetic oscillatory motion is shear-free.

The profiles of turbulent kinetic energy $\left[\bar{k}=1 / 2\left(\overline{u^{\prime 2}}+\overline{v^{\prime 2}}\right)\right]$ at several streamwise locations that include both the upstream and downstream regions are selectively shown in Fig. 4(a). When 
(a)

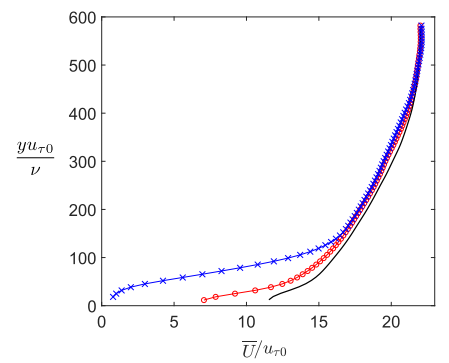

(b)

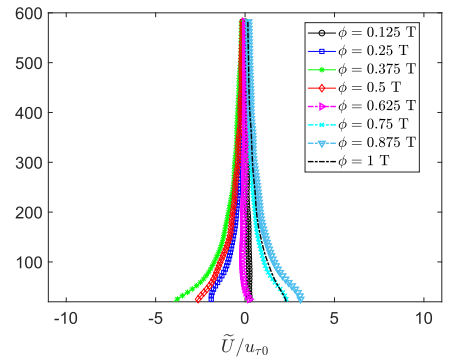

(c)

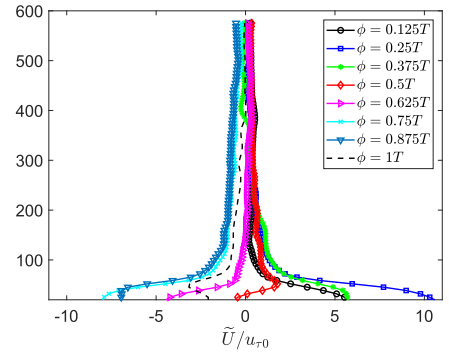

FIG. 3. Profiles of (a) $\bar{U}$ and (b) and (c) $\widetilde{U}$ in the (b) upstream and (c) downstream regions of the dynamic boundary array. In (a) diamonds denote upstream, crosses denote downstream, and the solid line shows the baseline case. In comparison with the upstream region, $\bar{U}$ decreases but $\widetilde{U}$ increases in the downstream region.

compared to the baseline case, $\bar{k}$ first increases in the upstream region and then decreases in the following downstream regions. The reduction of $\bar{k}$ near the wall happens together with the formation of an outer peak at a wall distance of $\frac{y u_{\tau 0}}{v}=80$. Figure 4(a) suggests that the turbulent activities are shifted away from the wall. Interestingly, the distribution of $\bar{k}$ closely follows the distribution of transverse vorticity $\left(\overline{\omega_{z}}=\frac{\partial \bar{V}}{\partial x}-\frac{\partial \bar{U}}{\partial y}\right)$ shown in Fig. 4(b). Figures 3 and 4 together depict the spatial distribution change of the energy components: The energetic turbulent motions are shifted away from the wall; in the near-wall region, the turbulence energy and the mean motion energy decrease, but the energy of shear-free motion increases.

\section{B. Energy exchange processes}

We analyze the energy exchange processes among the triple decomposed velocity components, which happen in the general three-dimensional space as shown by the tensor formula given in Fig. 1(b). With the current surface design and modulation strategy [densely packed dynamic surfaces, large transverse ( $z$ ) span, and in-phase modulation], the energy exchange activities (in the central region of the dynamic boundary array) are mainly confined in the $x-y$ plane. This assumption is validated by separated DPIV measurements in the $y-z$ and the $x-z$ plane located at several sampling locations. The out-of-plane components are at least an order of magnitude smaller than the $x-y$ plane components. Therefore, we approximate the energy exchange processes using their $x-y$ components only,

$$
\begin{aligned}
& \mathrm{TKP}_{\mathrm{MT}}=-\overline{u^{\prime} v^{\prime}} \frac{\partial \bar{U}}{\partial y}-\overline{u^{\prime} v^{\prime}} \frac{\partial \bar{V}}{\partial x}-\overline{u^{\prime 2}} \frac{\partial \bar{U}}{\partial x}-\overline{v^{\prime 2}} \frac{\partial \bar{V}}{\partial y}, \\
& \mathrm{TKP}_{\mathrm{MO}}=-\overline{\widetilde{U} \widetilde{V} \frac{\partial \bar{U}}{\partial y}}-\overline{\widetilde{U} \widetilde{V} \frac{\partial \bar{V}}{\partial x}}-\overline{\widetilde{U^{2}} \frac{\partial \bar{U}}{\partial x}}-\overline{\widetilde{V^{2}} \frac{\partial \bar{V}}{\partial y}} \\
& \mathrm{TKP}_{\mathrm{OT}}=-\overline{\left\langle u^{\prime} v^{\prime}\right\rangle \frac{\partial \widetilde{U}}{\partial y}}-\overline{\left\langle u^{\prime} v^{\prime}\right\rangle \frac{\partial \widetilde{V}}{\partial x}}-\overline{\left\langle u^{\prime 2}\right\rangle \frac{\partial \widetilde{U}}{\partial x}}-\overline{\left\langle v^{\prime 2}\right\rangle \frac{\partial \widetilde{V}}{\partial y}},
\end{aligned}
$$

where $u$ and $v$ represent the streamwise and wall-normal velocities, respectively, and $\langle\cdot\rangle$ represents the phase average.

We first analyze $\mathrm{TKP}_{\mathrm{MT}}$, the energy exchange between mean flow and turbulence. Figures 5(a) and 5(b) show the $\mathrm{TKP}_{\mathrm{MT}}$ profiles at two representative $x$ locations $\frac{x u_{\tau 0}}{v}=270$ and $\frac{x u_{\tau} 0}{v}=660$, where negative and positive $\mathrm{TKP}_{\mathrm{MT}}$ values are observed. The baseline TKP is shown as a reference, which is positive due to the dominant effect of turbulent shear production $-\overline{u^{\prime} v^{\prime}} \frac{\partial \bar{U}}{\partial y}$. The TKP $\mathrm{TT}_{\mathrm{MT}}$ at both $x$ locations is much larger than the baseline TKP, indicating the very active energy exchange 

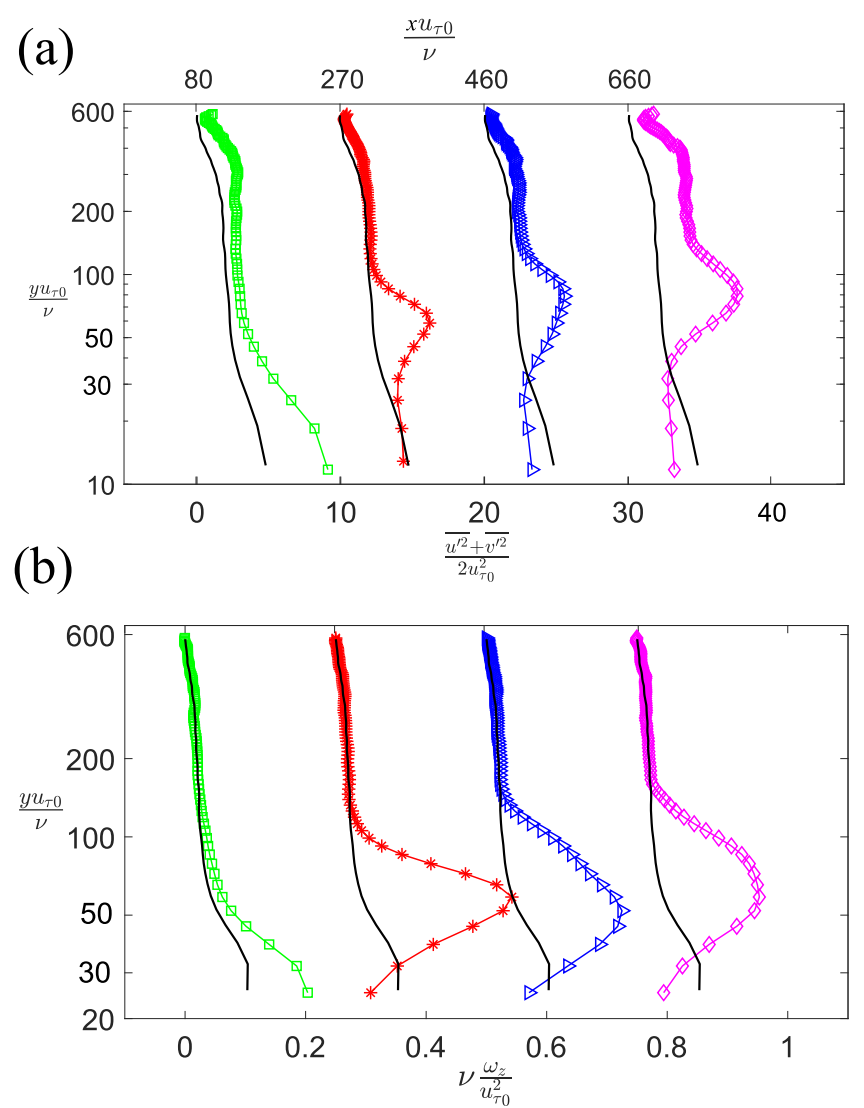

FIG. 4. Profiles of (a) turbulent kinetic energy $\bar{k}=1 / 2\left(\overline{u^{\prime 2}}+\overline{v^{\prime 2}}\right)$ and (b) transverse vorticity $\overline{\omega_{z}}$ exhibit similar evolution trends in the $x$ direction. Both $\bar{k}$ and $\overline{\omega_{z}}$ decrease near the wall and develop an outer peak. The streamwise locations $\left(\frac{x u_{\tau} 0}{v}\right)$ are given along the top edge of (a). The profiles of the baseline case are shown by the black solid line.

activities thereof. The dominant components of $\mathrm{TKP}_{\mathrm{MT}}$ are the shear production $-\overline{u^{\prime} v^{\prime}} \frac{\partial \bar{U}}{\partial y}$ and the normal production $-\overline{u^{\prime 2}} \frac{\partial \bar{U}}{\partial x}$. The shear production is positive $-\overline{u^{\prime} v^{\prime}} \frac{\partial \bar{U}}{\partial y}$ at both locations. However, its peak is shifted outward to the region of $\frac{y u_{\tau 0}}{v}=40-70$. In contrast, the normal production $-\overline{u^{\prime 2}} \frac{\partial \bar{U}}{\partial x}$ is very strong in the region below $\frac{y u_{t 0}}{v}=60$. The normal production is the dominant factor for TKP $\mathrm{MT}_{\mathrm{MT}}$ in the near-wall region.

Negative $-\overline{u^{\prime 2}} \frac{\partial \bar{U}}{\partial x}$ is observed in the relaminarizing TBLs, which is enabled by the favorable pressure gradient (or positive $\frac{\partial \bar{U}}{\partial x}$ ) [7,8]. However, the total TKP remains positive in that case, due to the dominant effect of the positive $-\overline{u^{\prime} v^{\prime}} \frac{\partial \bar{U}}{\partial y}$. As such, the relaminarization happens as a consequence of the turbulent dissipation surpassing the turbulent production $[7,8]$. The negative $\mathrm{TKP}_{\mathrm{MT}}$ in Fig. 5(a) represents a much stronger relaminarization effect, which suggests that the energy supply to turbulence (or the downward energy cascade) is reversed near the wall.

To demonstrate the general features of the three energy exchange processes, the contours of $\mathrm{TKP}_{\mathrm{MT}}, \mathrm{TKP}_{\mathrm{MO}}$, and $\mathrm{TKP}_{\mathrm{OT}}$ in the near-wall region are shown in Figs. 6(a), 6(b), and 6(c), respectively. The air films are represented by the green half-oval shapes along the bottom edge of each panel. Due to the inaccuracy of DPIV measurements in regions very close to the wall, the region below $\frac{y u_{\tau 0}}{v}=30$ is excluded from the contour plots. The three energy exchange contours 
(a)

(b)
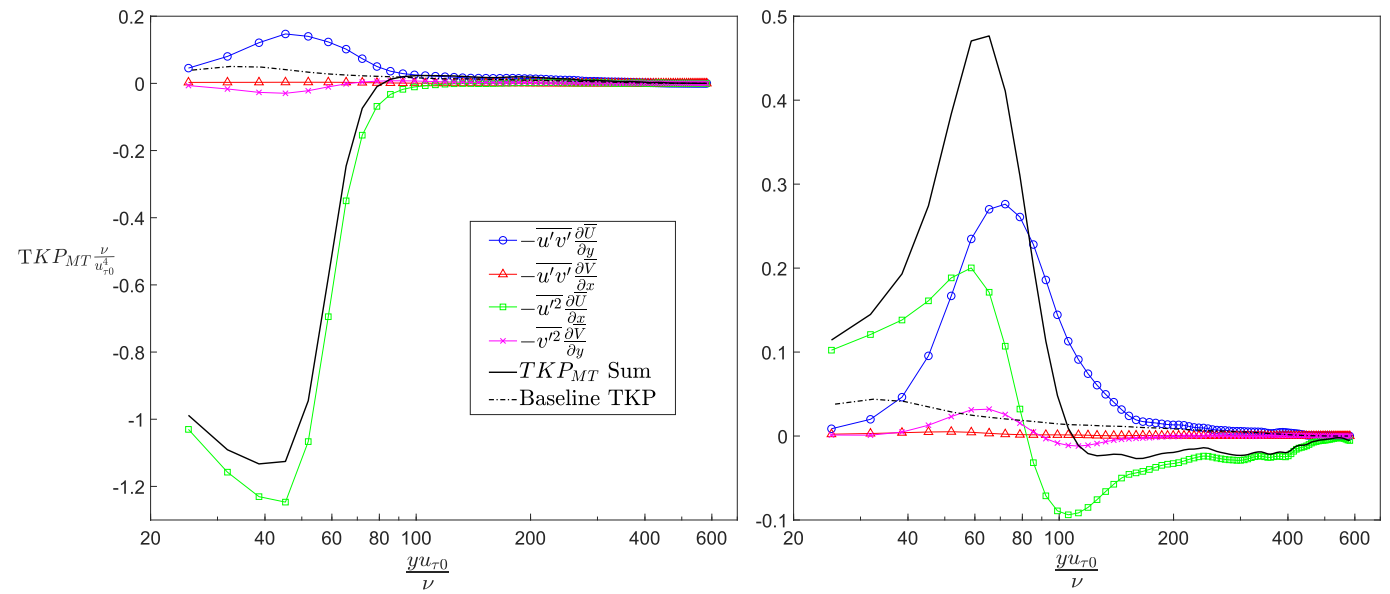

FIG. 5. Profiles of the individual components of $\mathrm{TKP}_{\mathrm{MT}}$ at two representative $x$ locations (a) $\frac{x u_{\tau} 0}{v}=270$ and (b) $\frac{x u_{\tau}}{v}=660$, where negative and positive values of $\mathrm{TKP}_{\mathrm{MT}}$ are observed. The baseline TKP is shown for reference. Note that the scale of the $y$ axis is different in the two panels.

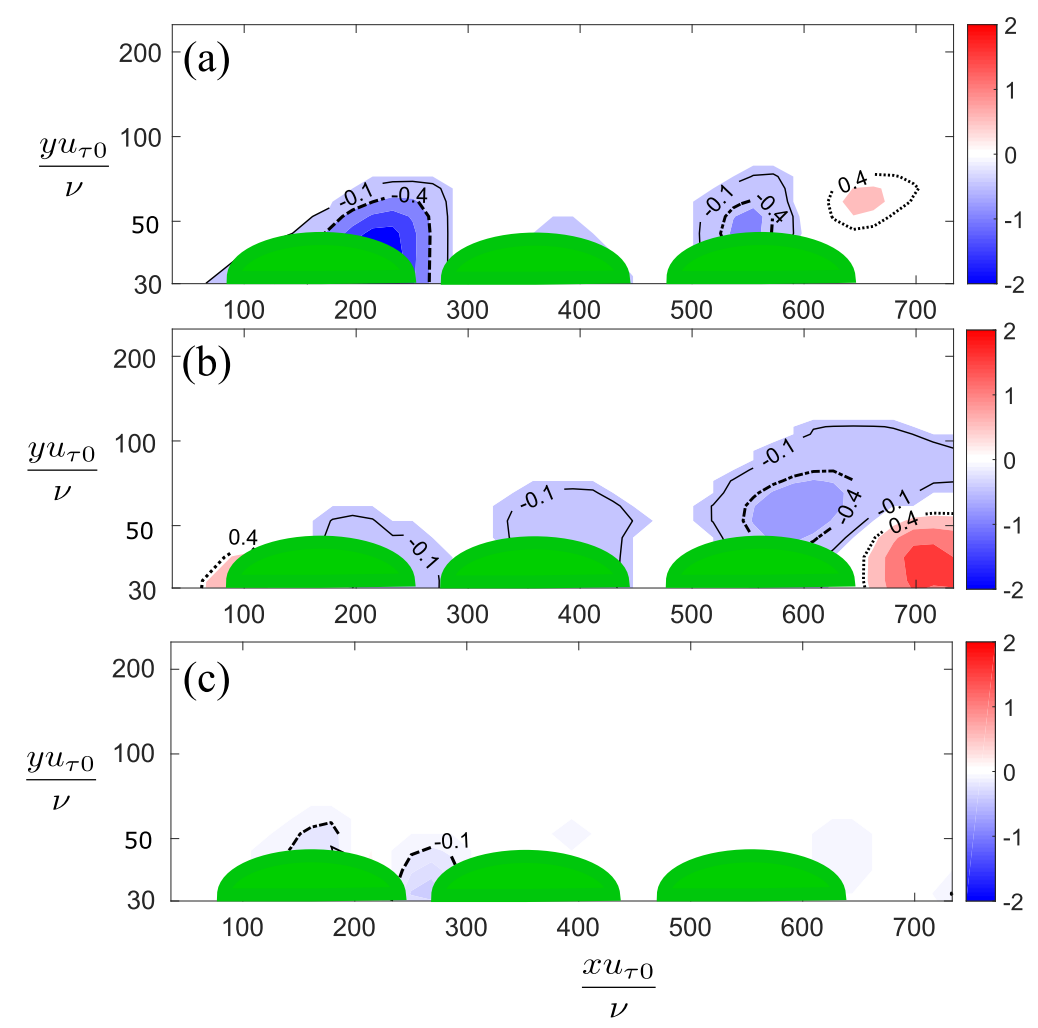

FIG. 6. Contours for (a) $\mathrm{TKP}_{\mathrm{MT}}$, (b) $\mathrm{TKP}_{\mathrm{MO}}$, and (c) $\mathrm{TKP}_{\mathrm{OT}}$ in the near-wall region. Air films are represented as green half ovals. 


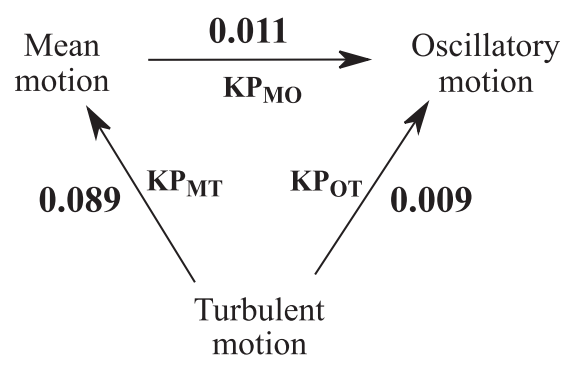

FIG. 7. Average value of the energy transfer process in the near-wall region between $\frac{y u_{\tau 0}}{v}=30$ and $\frac{y u_{\tau} 0}{v}=$ 65 and between $\frac{x u_{\tau 0}}{v}=10$ and $\frac{x u_{\tau 0}}{v}=750$. The direction of energy transfer is shown by the arrows. As a reference, the average value of the baseline TKP in the same region is 0.03 .

exhibit rapidly varying local features, i.e., they can change signs (the direction of energy transfer) in very short distances. The $\mathrm{TKP}_{\mathrm{MT}}$ and $\mathrm{TKP}_{\mathrm{MO}}$ are the two dominant energy exchange routes, while $\mathrm{TKP}_{\mathrm{OT}}$ is less significant. The $\mathrm{TKP}_{\mathrm{MT}}$ in Fig. 6(a) is negative (energy is transferred from turbulence to mean flow) in most regions except the downstream region of the air film array. The $\mathrm{TKP}_{\mathrm{MO}}$ in Fig. 6(b) is positive in the upstream or downstream region but is negative in other regions. The positive $\mathrm{TKP}_{\mathrm{MO}}$ implies energy supply to the oscillatory motion $\widetilde{U}$, which is in line with the strong $\widetilde{U}$ shown in Figs. 3(b) and 3(c). Interestingly, similar to $\mathrm{TKP}_{\mathrm{MT}}$, the major components of TKP $\mathrm{MO}$ are also the shear production $-\overline{\widetilde{U}} \widetilde{V} \frac{\partial \bar{U}}{\partial y}$ and the normal production $-\widetilde{\widetilde{U}}^{2} \frac{\partial \bar{U}}{\partial x}$. The involved velocity gradients $\frac{\partial \bar{U}}{\partial y}$ and $\frac{\partial \bar{U}}{\partial x}$ will be further analyzed in Sec. III C.

To examine the average effect of the highly localized energy exchange processes, a spatial average value for the near-wall region between $\frac{y u_{\tau} 0}{v}=30$ and $\frac{y u_{\tau} 0}{v}=65$ and between $\frac{x u_{\tau} 0}{v}=10$ and $\frac{x u_{\tau} 0}{v}=750$ is calculated for each of the contours in Fig. 6 . The data points very close to the air films are carefully removed to ensure the calculation accuracy. The spatial average value of the energy transfer process is summarized in Fig. 7, which suggests that the turbulent energy is transferred to both the mean motion and the oscillatory motion. In addition, energy is also transferred from the mean motion to the oscillatory motion. The direction of energy transfer is consistent with the spatial evolution trend of the velocity components presented in Figs. 3 and 4. Among the three energy transfer processes, the $\mathrm{TKP}_{\mathrm{MT}}$ is the major energy transfer route with an average value of 0.089 . As a reference, the average value of the baseline TKP in the same region is only 0.03 . Figure 7 suggests that the energy of turbulence is continuously transferred away. As such, we deduce that the turbulence in the near-wall region is relaminarized.

\section{Critical velocity gradients for the energy exchange process}

The energy transfer processes, as shown in Eq. (1), are the coproducts of local stresses (i.e., Reynolds stresses) and velocity gradients. Here we analyze the most important velocity gradients in the energy exchange processes, $\frac{\partial \bar{U}}{\partial y}$ and $\frac{\partial \bar{U}}{\partial x}$, because they contribute to the major components of both $\mathrm{TKP}_{\mathrm{MT}}$ and $\mathrm{TKP}_{\mathrm{MO}}$. The contours of $\frac{\partial \bar{U}}{\partial y}$ and $\frac{\partial \bar{U}}{\partial x}$ are shown in Figs. 8(a) and 8(b), respectively. Note that $\frac{\partial \bar{U}}{\partial y}$ is also the major constituent of the transverse vorticity $\overline{\omega_{z}}$ [shown in Fig. 4(b)], which typically reaches its maximum at the wall and sets the wall shear stress. In Fig. 8(a) the peak of $\frac{\partial \bar{U}}{\partial y}$ moves away from the wall. The distribution contour suggests the formation of two stationary vortices located above the free-slip boundaries. The normal velocity gradient $\frac{\partial \bar{U}}{\partial x}$ shown in Fig. 8(b) has a magnitude comparable to $\frac{\partial \bar{U}}{\partial y}$. Interestingly, the positive $\frac{\partial \bar{U}}{\partial x}$ is only observed in the region beneath the stationary vortices, which indicates a possible connection between the two quantities. 


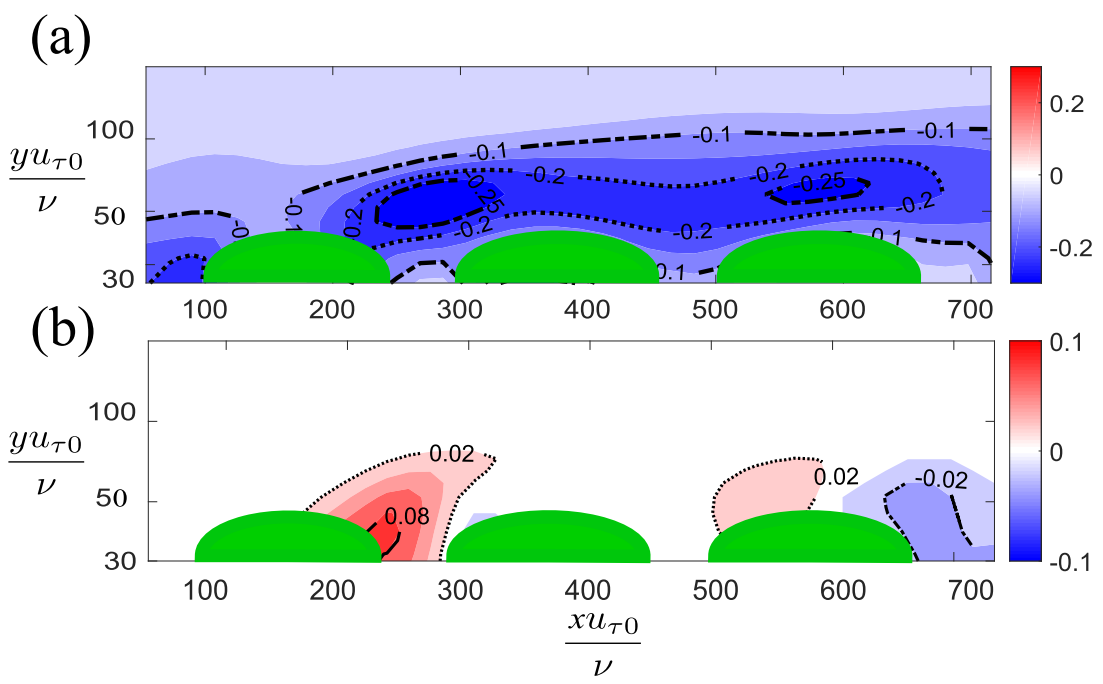

FIG. 8. Contours of the normalized velocity gradient (a) $\frac{\partial \bar{U}}{\partial y} \frac{v}{u_{\tau 0}^{2}}$ and (b) $\frac{\partial \bar{U}}{\partial x} \frac{\nu}{u_{\tau 0}^{2}}$. As the major component of $\overline{\omega_{z}}$, the distribution of $\frac{\partial \bar{U}}{\partial y}$ implies the existence of stationary vortices above the air film array. Beneath these vortices, strong positive $\frac{\partial \bar{U}}{\partial x}$ is generated.

The velocity gradients in Fig. 8 reflect the local flow structures to which the dominant energy exchange process $\mathrm{TKP}_{\mathrm{MT}}$ in Fig. 5 is due. Following the outward shift of the shear velocity gradient $\frac{\partial \bar{U}}{\partial y}$, the active (positive) turbulent shear production $-\overline{u^{\prime} v^{\prime}} \frac{\partial \bar{U}}{\partial y}$ happens in regions away from the wall. Meanwhile, the strong normal velocity gradient $\frac{\partial \bar{U}}{\partial x}$ in the near-wall region enables the nontrivial normal production $-\overline{u^{\prime 2}} \frac{\partial \bar{U}}{\partial x}$ thereof. The distribution pattern of positive (negative) $\frac{\partial \bar{U}}{\partial x}$ in Fig. 8(b) matches well with that of the negative (positive) $\mathrm{TKP}_{\mathrm{MT}}$ in Fig. 6(a), which demonstrates the dominant role of the normal production in the near-wall energy exchange process.

\section{DISCUSSION}

The presented relaminarization mechanism, as a much stronger effect than that in accelerated TBLs, can be briefly summarized as follows: The dynamic free-slip surfaces induces a strong shear-free oscillatory motion near the wall and effectively shifts the highly turbulent motions away from the wall. The spatial distribution of energy components and the energy transfer processes consistently support this mechanism. The relaminarization process seems a consequence of the lifted transverse vorticity and the depletion of near-wall shear motions that were reported in [9]. Following the outward shift of shear motions, the turbulent energy shear production, as the major energy supply of shear turbulence, is shifted away from the wall. As such, an outer peak and an inner reduction in the turbulent energy is observed in Fig. 4. In the near-wall region beneath the lifted high-shear motion, the energy transfer processes is essentially directed by the dominant unsteady oscillation thereof. As demonstrated in Fig. 5, the turbulent energy normal production overtakes the conventional shear production to be the major energy transfer mechanism near the solid surface. Interestingly, many of the reported observations, such as the reversed energy cascade and the formation of stationary vortices, were induced by nonlinear Faraday waves excited at a free water surface [11]. Because nonlinear surface waves essentially create a dynamic free-slip boundary, we deduce that the mean velocity gradient shown in Fig. 8 is linked to the nonlinear and nonsymmetric oscillation shown in Fig. 2. In this regard, some common physical mechanisms may be shared in the two different scenarios and deserve future attention. 
The detailed relaminarization mechanism is likely related to the vorticity generation and manipulation by the dynamic surface. It is known that a free-slip interface with a dynamic curvature can create vorticity and significantly influence the vorticity transportation process [12-14]. In this regard, the drastic curvature change of the dynamic free-slip boundary (shown in Fig. 1) can produce vorticity to interact with the near-wall vortical structures of the TBL. It is known that wall-bounded turbulence is self-sustained through an automatic cycle centering around the near-wall vortices, the interruption of which will lead to relaminarization [15]. In addition to vorticity generation, the current relaminarization can be related to the dynamic stretching effect caused by the unsteady acceleration near the wall. A relaminarization mechanism based on stretching the near-wall vortices was proposed in [16]. To verify the details and uncover the complete mechanism, more in-depth investigations will be required, such as through direct numerical simulations.

The presented relaminarization mechanism is limited in several aspects. First, its dependence on the oscillation frequency and amplitude is unclear. Second, in the energy exchange analysis, the out-of-plane components were not included. Although the out-of-plane components seem much smaller than the in-plane ones, their inclusion may lead to changes to the current conclusion. Finally, an analysis of the energy exchange across the dynamic surface (i.e., between the turbulent flow and the surface trapped air) is lacking, which also can contribute to the spatial evolution of the TBL. These concerns are left for future investigations.

\section{CONCLUSION}

We have demonstrated a local relaminarization mechanism induced by a dynamic free-slip boundary in the near-wall high-shear region of a TBL. Through the nonlinear interaction between the dynamic free-slip boundary and the TBL, the high-shear and high-turbulent motions are displaced away from the wall, with a shear-free oscillatory motion energized near the wall. Extraordinary phenomena, such as a locally reversed energy cascade, were observed. We presented results and analysis that support the strong drag reduction effect of the dynamic free-slip boundary from a perspective of energy exchange and transportation process. Existing in the most critical near-wall region, the relaminarization mechanism can be exploited for developing efficient drag control techniques.

\section{ACKNOWLEDGMENTS}

This work was supported by the Office of Naval Research under Grant No. N00014-15-1-2479. C.W. was supported by the Stanback Fellowship from the Graduate Aeronautical Laboratories of the California Institute of Technology, Caltech.

\section{APPENDIX A: ENERGY EXCHANGE PROCESS WITH REYNOLDS DECOMPOSITION}

With Reynolds decomposition, i.e., $U_{i}=\overline{U_{i}}+u_{i}^{\prime}$ and $P=\bar{P}+p^{\prime}$, the kinetic energy equations for the mean motion $\left(\frac{1}{2} \overline{U_{i} U_{i}}\right)$ and the turbulent motion $\left(\frac{1}{2} \overline{u_{i}^{\prime} u_{i}^{\prime}}\right)$ were derived in [3] and are given by

$$
\begin{aligned}
{\left[\frac{\partial}{\partial t}+\overline{U_{i}} \frac{\partial}{\partial x_{j}}\right]\left(\frac{1}{2} \overline{U_{i} U_{i}}\right)=} & \frac{\partial}{\partial x_{j}}\left\{-\frac{1}{\rho}\left(\overline{P U_{i}}\right) \delta_{i j}-\frac{1}{2}\left(\overline{u_{i}^{\prime} u_{j}^{\prime}}\right) \overline{U_{i}}+v\left(\left[\frac{\partial \overline{U_{i}}}{\partial x_{j}}+\frac{\partial \overline{U_{j}}}{\partial x_{i}}\right] \overline{U_{i}}\right)\right\} \\
& +\overline{\boldsymbol{u}_{i}^{\prime} \boldsymbol{u}_{j}^{\prime}} \frac{\partial \overline{\boldsymbol{U}_{i}}}{\partial \boldsymbol{x}_{j}}-\frac{1}{2} v\left(\frac{\partial \bar{U}_{i}}{\partial x_{j}}+\frac{\partial \bar{U}_{j}}{\partial x_{i}}\right)\left(\frac{\partial \overline{U_{i}}}{\partial x_{j}}+\frac{\partial \bar{U}_{j}}{\partial x_{i}}\right), \\
{\left[\frac{\partial}{\partial t}+\overline{U_{i}} \frac{\partial}{\partial x_{j}}\right]\left(\frac{1}{2} \overline{u_{i}^{\prime} u_{i}^{\prime}}\right)=} & \frac{\partial}{\partial x_{j}}\left\{-\frac{1}{\rho}\left(\overline{p^{\prime} u_{i}^{\prime}}\right) \delta_{i j}-\frac{1}{2}\left(\overline{u_{i}^{\prime} u_{i}^{\prime} u_{j}^{\prime}}\right)+v\left(\left[\frac{\partial u_{i}^{\prime}}{\partial x_{j}}+\frac{\partial u_{j}^{\prime}}{\partial x_{i}}\right] u_{i}^{\prime}\right)\right\} \\
& -\overline{\boldsymbol{u}_{i}^{\prime} \boldsymbol{u}_{j}^{\prime}} \frac{\partial \overline{\boldsymbol{U}_{\boldsymbol{i}}}}{\partial \boldsymbol{x}_{j}}-\frac{1}{2} v\left(\frac{\partial u_{i}^{\prime}}{\partial x_{j}}+\frac{\partial u_{j}^{\prime}}{\partial x_{i}}\right)\left(\frac{\partial u_{i}^{\prime}}{\partial x_{j}}+\frac{\partial u_{j}^{\prime}}{\partial x_{i}}\right)
\end{aligned}
$$


where the energy exchange between the mean motion and the turbulent motion, which is often referred to as turbulent kinetic energy production, is highlighted. Note that the TKP in the two equations adds up to zero. As such, the TKP only represents the energy transfer within the system, which does not increase or decrease the amount of total energy. Positive TKP means energy transfer from the mean motion to the turbulent motion and vice versa. The TKP in the $x-y$ plane is given by

$$
\mathrm{TKP}=\left(-\overline{u_{i}^{\prime} u_{j}^{\prime}}\right) \frac{\partial \bar{U}_{i}}{\partial x_{j}}
$$

In a flat plate TBL with zero pressure gradient, the TKP is usually dominated by the turbulent shear production $-\overline{u^{\prime} v^{\prime}} \frac{\partial \bar{U}}{\partial y}$, where $u$ and $v$ are the streamwise and wall-normal velocities, respectively.

\section{APPENDIX B: ENERGY EXCHANGE PROCESSES WITH TRIPLE DECOMPOSITION}

The energy equations with triple decomposition, e.g., $U_{i}=\bar{U}_{i}+\widetilde{U}_{i}+u_{i}^{\prime}$ and $P=\bar{P}+\widetilde{P}+p^{\prime}$, can be derived following the derivation process of Eqs. (A1) and (A2). The energy equations were derived in [17] and are given by

$$
\begin{aligned}
& {\left[\frac{\partial}{\partial t}+\bar{U}_{i} \frac{\partial}{\partial x_{j}}\right]\left(\frac{1}{2} \bar{U}_{i} \bar{U}_{i}\right)=-\frac{\partial \overline{P U}_{i}}{\partial x_{i}}-\left(-\overline{\boldsymbol{u}_{i}^{\prime} \boldsymbol{u}_{j}^{\prime}}\right) \frac{\partial \overline{\boldsymbol{U}}_{\boldsymbol{i}}}{\partial \boldsymbol{x}_{\boldsymbol{j}}}-\left(-\overline{\widetilde{\boldsymbol{U}}_{\boldsymbol{i}} \widetilde{U}_{\boldsymbol{j}}}\right) \frac{\partial \overline{\boldsymbol{U}}_{\boldsymbol{i}}}{\partial \boldsymbol{x}_{\boldsymbol{j}}}-\frac{\partial}{\partial x_{j}}\left[\bar{U}_{i}\left(\overline{u_{i}^{\prime} u_{j}^{\prime}}+\overline{\widetilde{U}_{i} \widetilde{U}_{j}}\right)\right]} \\
& +v \frac{\partial}{\partial x_{j}}\left[\bar{U}_{i}\left(\frac{\partial \bar{U}_{i}}{\partial x_{j}}+\frac{\partial \bar{U}_{j}}{\partial x_{i}}\right)\right]-\frac{1}{2} v\left(\frac{\partial \bar{U}_{i}}{\partial x_{j}}+\frac{\partial \bar{U}_{j}}{\partial x_{i}}\right)\left(\frac{\partial \bar{U}_{i}}{\partial x_{j}}+\frac{\partial \bar{U}_{j}}{\partial x_{i}}\right), \\
& {\left[\frac{\partial}{\partial t}+\overline{U_{i}} \frac{\partial}{\partial x_{j}}\right]\left(\frac{1}{2} \overline{\widetilde{U}_{i} \widetilde{U}_{i}}\right)=-\frac{\partial}{\partial x_{j}}\left[\overline{\widetilde{U}_{j}\left(\widetilde{P}+\frac{1}{2} \widetilde{U}_{i} \widetilde{U}_{i}\right)}\right]+\overline{\left(-\widetilde{\widetilde{U}_{i} \widetilde{U}_{j}}\right) \frac{\partial \overline{\boldsymbol{U}_{i}}}{\partial \boldsymbol{x}_{\boldsymbol{j}}}}} \\
& -\left(-\left\langle\boldsymbol{u}_{i}^{\prime} \boldsymbol{u}_{j}^{\prime}\right\rangle\right) \frac{\partial \widetilde{U}_{\boldsymbol{i}}}{\partial \boldsymbol{x}_{j}}-\frac{\partial}{\partial x_{j}} \overline{\left[\widetilde{U}_{i}\left\langle u_{i}^{\prime} u_{j}^{\prime}\right\rangle\right]}
\end{aligned}
$$

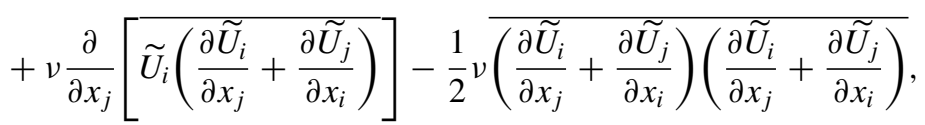

$$
\begin{aligned}
& {\left[\frac{\partial}{\partial t}+\overline{U_{i}} \frac{\partial}{\partial x_{j}}\right]\left(\frac{1}{2} \overline{u_{i}^{\prime} u_{i}^{\prime}}\right)=-\frac{\partial}{\partial x_{j}}\left[\overline{u_{j}^{\prime}\left(p^{\prime}+\frac{1}{2} u_{i}^{\prime} u_{i}^{\prime}\right)}\right]+\left(-\overline{\boldsymbol{u}_{i}^{\prime} \boldsymbol{u}_{j}^{\prime}}\right) \frac{\partial \overline{\boldsymbol{U}}_{\boldsymbol{i}}}{\partial \boldsymbol{x}_{\boldsymbol{j}}}} \\
& +\left(-\overline{\left\langle\boldsymbol{u}_{\boldsymbol{i}}^{\prime} \boldsymbol{u}_{j}^{\prime}\right\rangle \frac{\partial \widetilde{U}_{\boldsymbol{i}}}{\partial \boldsymbol{x}_{j}}}\right)-\overline{\widetilde{U}_{j} \frac{\partial}{\partial x_{j}}\left\langle\frac{1}{2} u_{i}^{\prime} u_{i}^{\prime}\right\rangle} \\
& +\frac{1}{2} v \frac{\partial}{\partial x_{j}} \overline{\left[u_{i}^{\prime}\left(\frac{\partial u_{i}^{\prime}}{\partial x_{j}}+\frac{\partial u_{j}^{\prime}}{\partial x_{i}}\right)\right]}-\frac{1}{2} v \overline{\left(\frac{\partial u_{i}^{\prime}}{\partial x_{j}}+\frac{\partial u_{j}^{\prime}}{\partial x_{i}}\right)\left(\frac{\partial u_{i}^{\prime}}{\partial x_{j}}+\frac{\partial u_{j}^{\prime}}{\partial x_{i}}\right)},
\end{aligned}
$$

where $\langle\cdot\rangle$ represents the phase average.

General energy exchange processes happen among the $\bar{U}_{i}, \widetilde{U}_{i}$, and $+u_{i}^{\prime}$, which are highlighted in the above equations. Similar to the TKP in the energy equations with Reynolds decomposition, the general energy exchange quantities add up to zero. The three different energy transfer routes are denoted by $\mathrm{TKP}_{\mathrm{MT}}$, $\mathrm{TKP}_{\mathrm{MO}}$, and $\mathrm{TKP}_{\mathrm{OT}}$, respectively, where $\mathrm{M}$ represents mean motion, $\mathrm{O}$ 
represents oscillatory motion, and T represents turbulent motion:

$$
\begin{aligned}
\mathrm{TKP}_{\mathrm{MT}} & =\left(-\overline{u_{i}^{\prime} u_{j}^{\prime}}\right) \frac{\partial \bar{U}_{i}}{\partial x_{j}}, \\
\mathrm{TKP}_{\mathrm{MO}} & =-\overline{\widetilde{U}_{i} \widetilde{U}_{j}} \frac{\partial \overline{U_{i}}}{\partial x_{j}}, \\
\mathrm{TKP}_{\mathrm{OT}} & =-\left\langle\overline{\left.u_{i}^{\prime} u_{j}^{\prime}\right\rangle \frac{\partial \widetilde{U}_{i}}{\partial x_{j}}} .\right.
\end{aligned}
$$

Approximated by their $x-y$ components, the three generalized energy exchange processes are given by

$$
\begin{aligned}
& \mathrm{TKP}_{\mathrm{MT}}=-\overline{u^{\prime} v^{\prime}} \frac{\partial \bar{U}}{\partial y}-\overline{u^{\prime} v^{\prime}} \frac{\partial \bar{V}}{\partial x}-\overline{u^{\prime 2}} \frac{\partial \bar{U}}{\partial x}-\overline{v^{\prime 2}} \frac{\partial \bar{V}}{\partial y}, \\
& \mathrm{TKP}_{\text {MO }}=-\overline{\widetilde{U} \widetilde{V} \frac{\partial \bar{U}}{\partial y}}-\overline{\widetilde{U} \widetilde{V} \frac{\partial \bar{V}}{\partial x}}-\overline{\widetilde{U^{2}} \frac{\partial \bar{U}}{\partial x}}-\overline{\widetilde{V^{2}} \frac{\partial \bar{V}}{\partial y}}, \\
& \mathrm{TKP}_{\text {Oт }}=-\overline{\left\langle u^{\prime} v^{\prime}\right\rangle \frac{\partial \widetilde{U}}{\partial y}}-\overline{\left\langle u^{\prime} v^{\prime}\right\rangle \frac{\partial \widetilde{V}}{\partial x}}-\overline{\left\langle u^{\prime 2}\right\rangle \frac{\partial \widetilde{U}}{\partial x}}-\overline{\left\langle v^{\prime 2}\right\rangle \frac{\partial \widetilde{V}}{\partial y}} \text {. }
\end{aligned}
$$

Note that $\mathrm{TKP}_{\mathrm{MT}}$ is identical to the TKP for the flat plate TBL given in Appendix A.

[1] K. Fukuda, J. Tokunaga, T. Nobunaga, T. Nakatani, T. Iwasaki, and Y. Kunitake, Frictional drag reduction with air lubricant over a super-water-repellent surface, J. Mar. Sci. Technol. 5, 123 (2000).

[2] I. Marusic, R. Mathis, and N. Hutchins, Predictive model for wall-bounded turbulent flow, Science 329, 193 (2010).

[3] H. Tennekes and J. L. Lumley, A First Course in Turbulence (MIT Press, Cambridge, 2018).

[4] R. Mukund, P. Viswanath, R. Narasimha, A. Prabhu, and J. Crouch, Relaminarization in highly favourable pressure gradients on a convex surface, J. Fluid Mech. 566, 97 (2006).

[5] R. Narasimha and K. Sreenivasan, in Advances in Applied Mechanics, edited by C.-S. Yih (Elsevier, Amsterdam, 1979), Vol. 19, pp. 221-309.

[6] K. Sreenivasan, Laminarescent, relaminarizing and retransitional flows, Acta Mech. 44, 1 (1982).

[7] D. Warnack and H. Fernholz, The effects of a favourable pressure gradient and of the Reynolds number on an incompressible axisymmetric turbulent boundary layer. Part 2. The boundary layer with relaminarization, J. Fluid Mech. 359, 357 (1998).

[8] C. Bourassa and F. Thomas, An experimental investigation of a highly accelerated turbulent boundary layer, J. Fluid Mech. 634, 359 (2009).

[9] C. Wang and M. Gharib, Effect of the dynamic slip boundary condition on the near-wall turbulent boundary layer, J. Fluid Mech. 901, A11 (2020).

[10] A. K. M. F. Hussain and W. C. Reynolds, The mechanics of an organized wave in turbulent shear flow, J. Fluid Mech. 41, 241 (1970).

[11] N. Francois, H. Xia, H. Punzmann, and M. Shats, Inverse Energy Cascade and Emergence of Large Coherent Vortices in Turbulence Driven by Faraday Waves, Phys. Rev. Lett. 110, 194501 (2013).

[12] G. Batchelor, An Introduction to Fluid Dynamics (Cambridge University Press, Cambridge, 2000).

[13] H. G. Hornung, C. Willert, and S. Turner, The flow field downstream of a hydraulic jump, J. Fluid Mech. 287, 299 (1995).

[14] M. Gharib and A. Weigand, Experimental studies of vortex disconnection and connection at a free surface, J. Fluid Mech. 321, 59 (1996).

[15] J. Jiménez and A. Pinelli, The autonomous cycle of near-wall turbulence, J. Fluid Mech. 389, 335 (1999). 
[16] G. L. Brown, M. Lee, and R. D. Moser, TSFP Digital Library Online (Begell House, Danbury, 2015).

[17] W. Reynolds and A. Hussain, The mechanics of an organized wave in turbulent shear flow. Part 3. Theoretical models and comparisons with experiments, J. Fluid Mech. 54, 263 (1972). 